\title{
Avaliação de fibrinogênio, tempo de tromboplastina parcial e tempo de protrombina em pacientes com infarto agudo do miocárdio
}

\author{
Evaluation of fibrinogen, activated partial thromboplastin time and prothrombin time in patients with acute \\ myocardial infarction
}

Marinês Lavall Dias', José Edson Paz da Silva², Aline B. Wohlfahrt' ${ }^{3}$, Natália Brucker ${ }^{3}$

\begin{abstract}
unitermos resumo
Fibrinogênio

Infarto agudo do miocárdio

Este trabalho procurou ressaltar a importância de parâmetros laboratoriais como marcadores de risco no infarto agudo do miocárdio (IAM). O IAM é um dos maiores problemas de saúde pública no mundo, sendo que dois terços dos pacientes que buscam atendimento emergencial não têm causa cardíaca, não

Tempo de protrombina havendo necessidade de hospitalização. Concentrações de fibrinogênio medidas durante a fase aguda do IAM foram associadas a morte cardiovascular ou novo evento de IAM. A incidência de eventos foi maior em homens na faixa etária de 44 a 75 anos e nas mulheres entre 56 a 90 anos. Aproximadamente $73 \%$ dos pacientes apresentavam história familiar de doença arterial coronariana (DAC), 66\% fumavam, $63 \%$ apresentavam hipertensão e a maioria era sedentária. Foi observado que nos dias frios ou com temperaturas extremas houve maior número de infartados. Para as concentrações de fibrinogênio (Fbr), tempo de protrombina (TP), troponina (TROP), creatinoquinase (CK), fração $M B$ da creatinoquinase (CK-MB) e contagem de leucócitos, a média dos resultados obtidos apresentou diferença significativa entre os grupos. No entanto, para tempo parcial de tromboplastina ativada (TTPa), colesterol total, lipoproteína de alta densidade (HDL), lipoproteína de baixa densidade (LDL) e triglicérides, as médias observadas não apresentaram diferença significativa entre o grupo controle e infartados. Neste trabalho foi possível demonstrar uma tendência de aumento crescente da concentração de fibrinogênio nos pacientes, que se mostrou adequada como um dos marcadores laboratoriais para IAM.
\end{abstract}

abstract

We focused on the importance of laboratorial parameters as risk factors for acute myocardial infarction (AMI). $A M I$ is one of the major problems of public health in the world. However, approximately two thirds of patients that attend emergency rooms do not have any cardiac injury or life risk illness, therefore not requiring to be hospitalized. Fibrinogen concentrations measured during the acute phase of AMI were related to cardiovascular death or a new AMI event. This incidence was higher in the age range of 44 to 75 years in men, and 56 to 90 in women. Approximately $73 \%$ of patients presented family history of coronary heart disease (CHD), 66\% were smokers, $63 \%$ presented hypertension and most of them were sedentary. Increased incidence of AMI in extreme temperatures was also observed. For fibrinogen concentrations (Fbr), results demonstrated significant difference $(p<0.05)$ between control and AMI patients. Considering troponin $(T R O P)$, creatine kinase $(C K)$, the $M B$ fraction of creatine kinase (CK-MB) and leukocytes count, results showed statistically significant differences. However, partial thromboplastin time activated (PTAT), total cholesterol (TC), high-density lipoprotein (HDL), low-density lipoprotein $(L D L)$, and triglyceride levels presented no significant difference between the studied groups. In conclusion, this work demonstrated a trend towards increasing fibrinogen concentration in patients with AMI, revealing that it may be considered one of the cardiac markers for AMI. key words

Fibrinogen

Acute myocardial infarction

Prothrombin time

1. Farmacêutica bioquímica da Universidade Federal de Santa Maria (UFSM)

2. Professor-doutor adjunto do Departamento de Análises Clínicas e Toxicológicas da UFSM.

3. Acadêmica do Curso de Farmácia da UFSM.

Trabalho realizado no Departamento de Análises Clínicas e Toxicológicas e apresentado como dissertação de mestrado em janeiro de 2006 na UFSM. 


\section{Introdução}

Um dos maiores problemas de saúde pública no mundo é o infarto agudo do miocárdio (IAM). Em 1912, Herrick descreveu o primeiro caso, que levantou a hipótese de oclusão coronariana por trombo. As patologias cardíacas de origem aterosclerótica tornaram-se um dos grandes males do século no Brasil e na maioria dos países desenvolvidos ${ }^{(3)}$. A maioria dos casos de IAM é causada pela aterosclerose ${ }^{(20)}$. Existem fatores inalteráveis que contribuem para essa patologia: idade avançada, sexo e predisposição genética. Os principais fatores de risco que levam à aterosclerose são dislipidemia, hipertensão arterial sistêmica (HAS) e tabagismo. Há outros fatores de risco, como sedentarismo, obesidade, homocisteinemia, consumo de álcool, fatores psicológicos, contraceptivos orais e menopausa ${ }^{(1)}$.

No Brasil, os dados do Sistema Único de Saúde (SUS) indicaram 1,1 milhão de internações por doença do aparelho circulatório em 1999, sendo 133 mil por doença isquêmica do coração. Os custos para o SUS foram de R\$763 milhões e R\$203 milhões, respectivamente, o que representa aproximadamente $4,2 \%$ e $1,1 \%$ do orçamento daquele ano do Ministério da Saúde.

Dois terços dos pacientes que procuram atendimento emergencial não o fazem por causa cardíaca ou doença com risco de vida. Mesmo assim, a maioria desses pacientes é hospitalizada para investigação diagnóstica ${ }^{(19)}$. Devido a isso, torna-se importante encontrar marcadores laboratoriais de qualidade e menor custo. As taxas de IAM não-diagnosticados, liberados para retornarem ao seu domicílio, são de $2,1 \%$. A mortalidade em pacientes com IAM que são liberados inapropriadamente está em torno de $25 \%$, quase o dobro daqueles internados ${ }^{(16)}$.

Diversos estudos realizados na última década demonstraram a possibilidade de encontrar marcadores laboratoriais de qualidade, desde que sejam utilizadas estratégias diagnósticas que acelerem o processo assistencial e que tenham elevadíssima acurácia, reduzindo significativamente não só o tempo de permanência hospitalar como também a taxa de internação desnecessária de pacientes em unidades de alta complexidade(4).

O diagnóstico precoce do IAM tem importância crítica para a sobrevivência do paciente. Cerca de $50 \%$ dos pacientes com IAM apresentam em seu ingresso em urgências um eletrocardiograma (ECG) não-diagnóstico, sugerindo a necessidade de dispor de marcadores cardíacos específicos e sensíveis. Com o objetivo de encontrar esses marcadores para o diagnóstico do IAM, este trabalho de pesquisa analisou alguns parâmetros laboratoriais e também alguns fatores de risco para IAM.

\section{Material e método}

Foram realizados um estudo caso-controle e a coleta de dados prospectivos. Os grupos de pacientes com IAM foram selecionados no pronto-atendimento (PA) da Avenida Medianeira de Santa Maria (RS) e no ambulatório do Hospital Universitário de Santa Maria (PA do HUSM), que atendem pacientes de toda a região central do estado. Esses locais foram eleitos para a realização do estudo por serem locais de emergência onde o paciente é primeiramente atendido. Desse modo, conseguimos amostras sangüíneas antes da terapêutica farmacológica. A população de estudo envolveu indivíduos com IAM e indivíduos saudáveis. O estudo foi realizado no período de novembro de 2004 a setembro de 2005, e foram estudados 54 participantes (32 pacientes e 22 controles).

\section{Critérios de inclusão para casos}

Pacientes com diagnóstico de IAM confirmado pela anamnese do paciente, alterações eletrocardiográficas e aumento dos níveis séricos da fração $\mathrm{MB}$ da creatinoquinase (CK-MB) e/ou creatinoquinase (CK) e troponina (TROP).

\section{Critérios de exclusão para casos}

Pacientes sem diagnóstico de IAM; aqueles que faziam uso de antiagregante plaquetário, anticoagulantes (warfarina sódica ou heparina) ou que tinham recebido terapêutica trombolítica antes da coleta do sangue para os testes da coagulação. As amostras de sangue foram colhidas imediatamente antes do início da terapêutica específica para evitar resultados que prejudicassem a análise do estudo.

\section{Critérios de inclusão para controles}

Indivíduos sadios do ponto de vista clinicolaboratorial, com idade e sexo semelhantes aos dos pacientes infartados. Para a comprovação da ausência de doenças nos indivíduos do grupo controle, foram realizados exames laboratoriais.

\section{Critérios de exclusão para controles}

Diagnóstico prévio de doença arterial coronariana (DAC), diabetes, hepatopatias, neoplasias ou qualquer outra patologia que, a critério do investigador, pudesse impedir a inclusão ou prejudicar a análise do estudo; utilização de medicamento que viesse a alterar os resultados dos exames laboratoriais. 


\section{Variáveis estudadas}

\section{Variáveis relacionadas à identificação}

Idade, sexo, tipagem sangüínea.

\section{Variáveis relacionadas ao meio ambiente}

Temperatura ambiente e umidade do ar de Santa Maria durante o período de estudo. Os dados foram obtidos das fichas que estão arquivadas no Departamento de Fitotecnia da Universidade Federal de Santa Maria (UFSM).

\section{Variáveis relacionadas com o estilo de vida}

Consumo de bebidas alcoólicas, atividade física, tabagismo, estresse, história familiar de IAM.

\section{Coleta de dados}

Foi realizado contato inicial com os médicos ou enfermeiros para obter informações sobre internação de pacientes com suspeita de IAM e então foi coletada a amostra biológica, levada imediatamente ao laboratório clínico para realização dos exames. Mediante consulta ao prontuário do paciente, confirmou-se o diagnóstico de IAM e dirigiu-se ao paciente e/ou ao acompanhante para realização do questionário e obtenção do consentimento livre e esclarecido (pós-informado).

\section{Casuística}

Dos 32 pacientes infartados, 21 eram do sexo masculino (65\%) e 11 (35\%), do sexo feminino. A idade média foi de 66 anos, sendo que $43 \%$ do total de pacientes tinham mais de 70 anos de idade. A hipertensão estava presente em $63,3 \%$ dos pacientes (Figura 1). Dor torácica foi um sintoma prevalente, seguido de dispnéia, tosse, náuseas, tontura, tremor, frio e vômito.

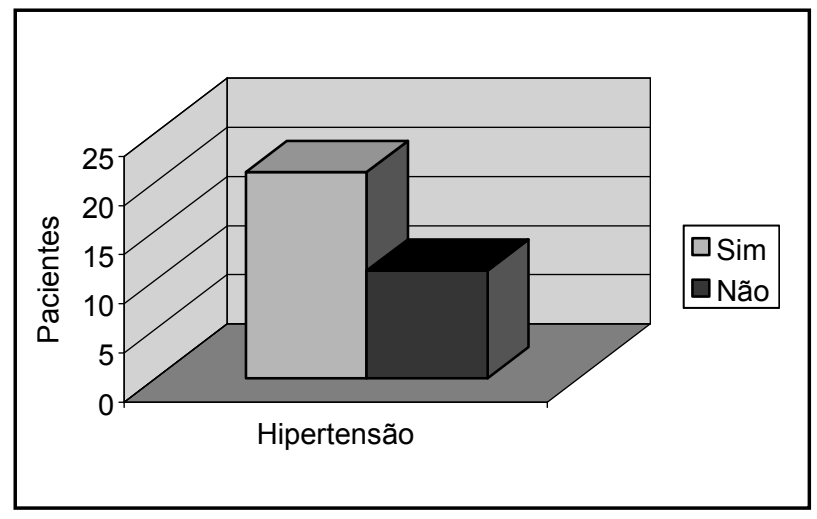

Figura 1 - Distribuição dos pacientes com hipertensão

\section{Metodologia}

De 32 pacientes e 22 controles, por meio do sistema Vacutainer ${ }^{\circledR}$ em tubos apropriados para a realização dos exames, amostras de aproximadamente $10 \mathrm{ml}$ de sangue venoso foram coletadas e analisadas no laboratório de análises clínicas do HUSM. A coleta de sangue foi realizada logo na chegada do paciente ao ambulatório. As amostras foram imediatamente processadas e os seguintes parâmetros foram medidos: hemograma, tempo de protrombina (TP), tempo de tromboplastina parcial (TTPa), fibrinogênio (Fbr), colesterol total (CT) e frações, gama-glutamiltransferase (gama-GT), triglicérides, CK, CK-MB e TROP.

Com o fim de evitar interferências nos resultados dos exames, a amostra foi coletada na chegada do paciente ao pronto-atendimento, antes que ele fosse medicado.

A determinação dos níveis plasmáticos de fibrinogênio foi realizada por método descrito por Clauss (1957), turbidimétrico, utilizando o equipamento Coag A Mate MTX (Organon Teknika ${ }^{\circledR}$ ). O valor de referência é de $148-380$ $\mathrm{mg} / \mathrm{dl}$ em indivíduos normais.

TP com ISI 1,17 e padrão 13,08; TTPa e atividade realizados no equipamento Coag A Mate MTX (Organon Teknika ${ }^{\circledR}$. O hemograma foi feito no equipamento Couter STKS; os exames bioquímicos no Hitachi ${ }^{\mathrm{TM}}$ (Roche $\mathrm{SA}$ ), pelo método de química úmida. A dosagem da TROP, utilizando equipamento Immulite ${ }^{\mathrm{TM}}$, foi determinada por quimioluminescência. Os resultados dos exames laboratoriais foram buscados no laboratório ou na pasta do paciente que se encontrava no PA ou no Serviço de Arquivo Médico e Estatístico (SAME) do HUSM.

Todos os testes foram realizados no Laboratório de Análises Clínicas (LAC) em sistemas automatizados, seguindo as instruções dos fabricantes.

\section{Estatística}

Para a análise estatística foi empregado o programa Statistical Analysis System, versão 8.02. Os dados foram inicialmente submetidos a uma análise descritiva para a obtenção de médias e desvios padrões. Para testar a existência ou não de diferença significativa entre os grupos para as variáveis estudadas, foi aplicado um teste de normalidade, constatando a não-normalidade das variáveis; logo se utilizou um teste não-paramétrico: Kruskal Wallis. O nível de significância utilizado foi de 5\%.

\section{Aspectos éticos}

O projeto foi aprovado pelo Comitê de Ética em Pesquisa do HUSM. 


\section{Resultados}

Neste estudo foram avaliados parâmetros hemostáticos que investigam o grau de hipercoagulabilidade em situações clínicas nas quais ocorre exacerbação da ativação do mecanismo da coagulação.

Verificou-se maior incidência de eventos em homens na faixa etária de 44 a 75 anos; as mulheres estavam na faixa etária entre 56 e 90 anos. Foi observado predomínio do grupo sangüíneo A entre os pacientes (Figura 2). A maioria não praticava exercícios físicos regularmente (Figura 3); $55,5 \%$ dos pacientes fumantes apresentaram fibrinogênio normal (Figura 4).

Os pacientes foram questionados quanto à presença, na família, de fatores de risco como HAS e DAC, com 76,6\% e $73,3 \%$ apresentando, respectivamente, esses fatores.

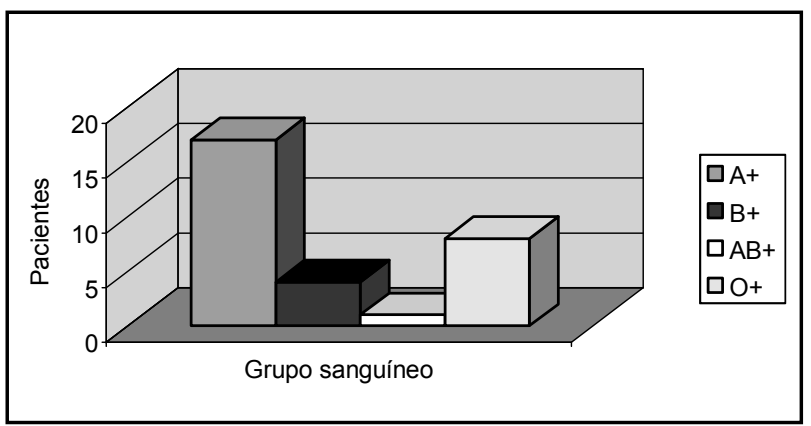

Figura 2 - Distribuição dos pacientes por grupo sangüíneo

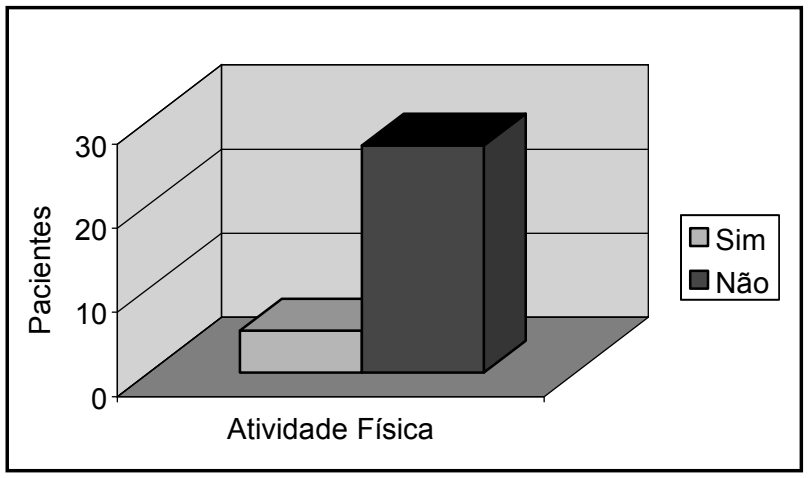

Figura 3 - Distribuição dos pacientes em relação à atividade física

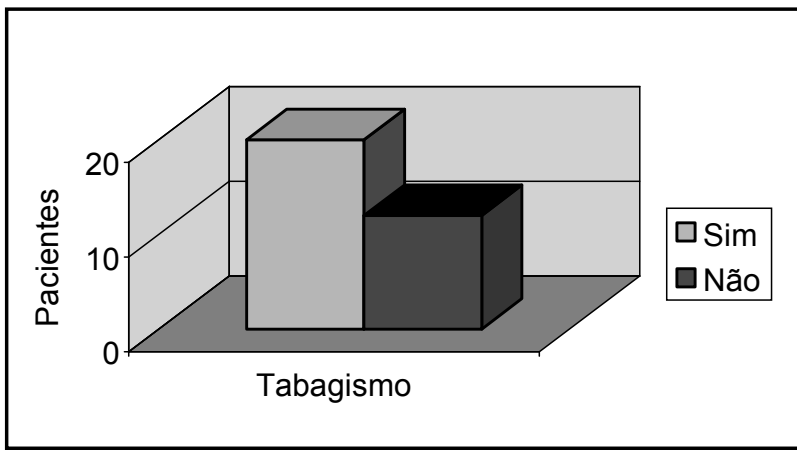

Figura 4 - Distribuição dos pacientes em relação ao fumo

A média e o respectivo desvio padrão de cada parâmetro analisado, como Fbr, TP, TTPA, contagem de leucócitos, hemoglobina $(\mathrm{Hb})$, hematócrito $(\mathrm{Ht}), \mathrm{CT}, \mathrm{HDL}$, troponina, CK, CK-MB, em amostras de sangue dos grupos controle e com infarto podem ser observados na Tabela 1.

Para as concentrações de Fbr, as médias observadas apresentaram diferença significativa $(p<0,05)$ entre o grupo controle e infartados, porém os níveis permaneceram dentro dos valores de referência para normalidade (Tabela 1).

Para o TP, a média dos resultados obtidos apresentou diferença significativa $(p<0,001)$ entre os grupos. $O$ fato pode ser devido ao uso de medicamentos, como antiinflamatórios, ácido acetilsalicílico, e outros que podem alterar a coagulação sangüínea, ou a algum procedimento incorreto na coleta da amostra.

Houve diferença significativa entre o grupo controle e infartados para as determinações de fibrinogênio, gamaGT, TP, leucometria, neutrófilos, TROP, CK-MB (58\% dos pacientes com valores elevados) e CK $(p<0,05)$ entre os grupos; $51 \%$ dos pacientes apresentavam CK acima da taxa de normalidade (Tabela 1).

A hipertensão estava presente em 63,3\% dos pacientes.

Dos pacientes com IAM, 67\% eram ansiosos (Figura 5).

A maioria dos pacientes informou não consumir bebidas alcoólicas (Figura 6).

Média dos valores encontrados nas determinaçōes de fibrinogênio (Fbr), contagem total de leucócitos (leuc), creatinoquinase (CK), (CK-MB), troponina (TROP), tempo de protrombina

Tabela 1 (TP), tempo de tromboplastina parcial (ITPa)

\begin{tabular}{lccccccc}
\hline & Fbr & leuc & CK & CK-MB & TROP & IP & ITPa \\
Controle & 233,8 & 8.009 & 98,63 & 20,3 & 0,1 & 13,83 & 27,1 \\
Infartado & $324,68^{*}$ & $12.810^{* *}$ & $266,57^{*}$ & $54,85^{*}$ & $11,52^{* *}$ & $16,51^{* *}$ & $29,67^{* * *}$ \\
\hline
\end{tabular}

${ }^{*} p<0,05 ;{ }^{* *} p<0,001 ;{ }^{* *}$ não-significativo. 


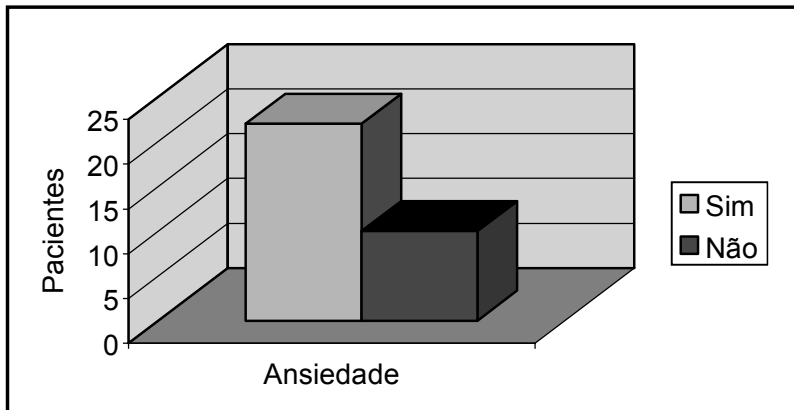

Figura 5 - Distribuição dos pacientes relacionada com a ansiedade

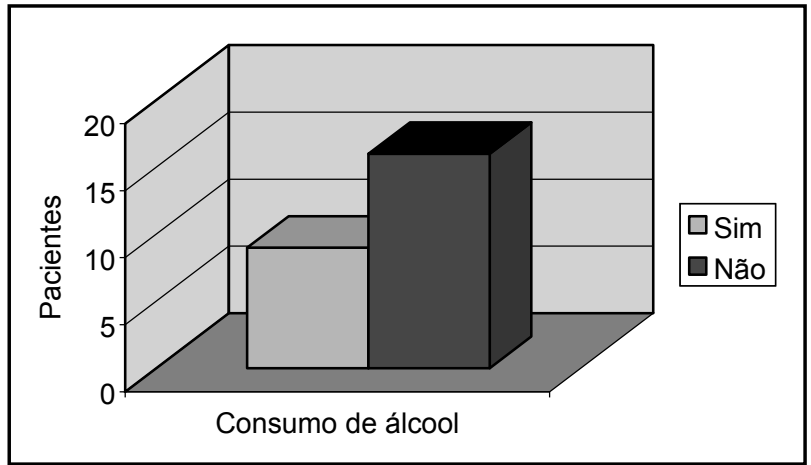

Figura 6 - Distribuição dos pacientes em relação ao consumo de álcool

Para $\mathrm{Ht}$ e plaquetas, a média dos resultados obtidos não apresentou diferença significativa entre os grupos (Tabela 1).

A enzima gama-GT apresentou-se elevada em 52\% dos pacientes.

Para CT, LDL, HDL, Hb, Ht, plaquetas e TTPa as médias observadas não apresentaram diferença significativa $(p>$ $0,05)$ entre o grupo controle e infartados. Verificou-se que $50 \%$ dos pacientes estavam com colesterol total acima de $200 \mathrm{mg} / \mathrm{dl}$.

O mês de julho apresentou maior incidência dos casos de infarto, 24,4\% dos pacientes; a temperatura média do mês foi aproximadamente $15^{\circ} \mathrm{C}$ e a umidade, $76,45 \%$, sendo a mínima $7,35^{\circ} \mathrm{C}$ e a máxima, $27,5^{\circ} \mathrm{C}$ (Tabela 2 ).

\section{Discussão}

Para o TP, a média dos resultados obtidos apresentou diferença significativa $(p<0,001)$ entre os grupos. Esse fato pode ser explicado por alterações nos fatores da via extrínseca devido ao uso de medicamentos, como antiinflamatórios, ácido acetilsalicílico, ou coleta de quantidade insuficiente de sangue, no tubo, em relação ao anticoagulante, que pode alterar TP e TTPa.
Relação do número de casos de IAM com a temperatura média e a

Tabela 2 umidade do dia

Mês Temperatura Umidade

Casos média ${ }^{\circ} \mathrm{C}$ média

$\begin{array}{lccc}\text { Novembro } & 21,48 & 71,5 & 1 \\ \text { Dezembro } & 24,1 & 63,8 & 4 \\ \text { Janeiro } & 26,6 & 65,1 & 3\end{array}$

Janeiro

65,1

Fevereiro

25,1

73

7

Março

24,58

61,1

20,08

81,95

18,6

82,45

18,68

82,65

15

76,45

18

70,45

Agosto

Total

41

Além dos casos relacionados na casuística deste trabalho foram incluídos na análise nove casos de pacientes com IAM que chegaram ao PA do HUSM.

As médias de $\mathrm{CT}, \mathrm{LDL}, \mathrm{HDL}, \mathrm{Hb}, \mathrm{Ht}$, plaquetas e TTPa não apresentaram diferença significativa $(p>0,05)$ entre o grupo controle e infartados. Verificou-se que $50 \%$ dos pacientes estavam com colesterol total acima de $200 \mathrm{mg} / \mathrm{dl}$, confirmando estudos que demonstraram que a vasta maioria de pacientes que desenvolvem doença cardíaca apresenta níveis lipídicos normais ou moderadamente elevados ${ }^{(18)}$.

Uma análise global dos dados apresentados na tabela permite concluir que no IAM há tendência à maior ativação do mecanismo da coagulação. Os níveis mais elevados de fibrinogênio nesses pacientes, comparando-se com o grupo controle $(p<0,05)$, sugerem que o mecanismo da coagulação está ativado, predispondo à formação do trombo (Tabela 1).

Foi relatado que o aumento nos níveis plasmáticos de fibrinogênio está associado à doença coronariana, o que justifica a tendência a se elevarem nesses pacientes ${ }^{(12)}$.

Uma comparação dos resultados dos parâmetros hemostáticos permite dizer que o encontro de níveis elevados de fibrinogênio assume grande importância na definição desse perfil nos pacientes estudados quando comparados aos controles. Portanto, os resultados encontrados suportam a relevância das alterações hemostáticas na fisiopatologia do IAM, considerando que a presença e a intensidade dessa 
alteração estão intimamente relacionadas com o agravamento da forma clínica.

Os resultados do TP foram altamente significativos, podendo ser explicados por alterações nos fatores da via extrínseca. Atualmente, esses parâmetros não têm sido valorizados, mas mostraram que em pacientes com reinfarto o TP e TTPa apresentaram-se elevados, confirmando a importância de avaliar a função hemostática nos pacientes com fatores de risco para IAM(10, 15). TP e TTPa ficaram prolongados por 36 horas, com um máximo nas primeiras horas após a lise. Após 24 a 36 horas há tendência de normalização da hemostasia.

Estudos indicam que a cascata da coagulação sangüínea está ativada em pacientes com aterosclerose periférica ${ }^{(9)}$. No entanto, a maioria desses pacientes utilizava algum tipo de medicamento que pode ter elevado o TP ou algum procedimento incorreto na hora da coleta da amostra.

O TTPa é o teste de triagem para avaliação dos fatores de coagulação das vias intrínseca e comum. Um TTPa alongado indica uma possível deficiência de fatores da coagulação ou a presença de inibidores ${ }^{(13)}$. No entanto, o TTPa não estava elevado nesses pacientes, demonstrando que os fatores de coagulação da via intrínseca estão normais (Tabela 1).

Para a CK, a média dos resultados obtidos apresentou diferença significativa $(p<0,05)$ entre os grupos (Tabela 1$)$, o mesmo ocorrendo com a CK-MB (Tabela 1).

Dos pacientes com IAM, 67\% eram ansiosos. Vários estudos afirmam que pessoas portadoras de personalidade ansiosa ou depressiva (Figura 7) são mais propensas a problemas coronarianos do que as não-portadoras. Existem muitas evidências de que o estresse social aumenta o risco de $\operatorname{IAM}^{(2)}$.

O hábito de praticar exercícios físicos regulares previne doenças cardiovasculares, combate o sedentarismo e estimula hábitos saudáveis ${ }^{(5)}$. A ausência de exercícios físicos regulares justifica em parte o IAM na maioria desses pacientes (Figura 3).

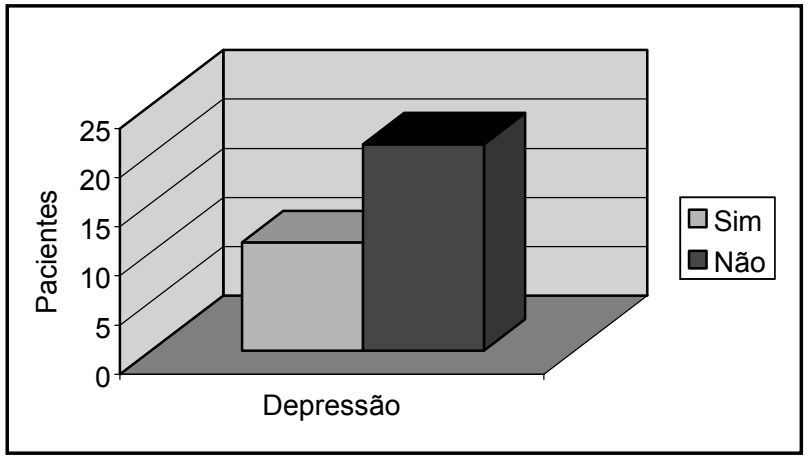

Figura 7 - Distribuição dos pacientes relacionada com a depressão
Apesar de a maioria desses pacientes ter informado não consumir bebidas alcoólicas (Figura 5), estudos anteriores demonstraram que o álcool pode causar lesão no músculo cardíaco. Entretanto existem estudos sugestivos de que pequenas doses de bebida alcoólica podem prevenir o IAM ${ }^{(22)}$.

Na Tabela 2 observa-se que o mês de julho apresentou maior incidência dos casos de infarto: $24,4 \%$ dos pacientes. A temperatura média do mês foi aproximadamente $15^{\circ} \mathrm{C}$ e a umidade, $76,45 \%$; sendo a mínima $7,35^{\circ} \mathrm{C}$ e a máxima, $27,5^{\circ} \mathrm{C}$. Isso está de acordo com estudos anteriores nos quais maior número de hospitalizações por IAM ocorre nos meses de inverno ${ }^{(14)}$. Nos dias com extremos de temperatura houve $30 \%$ mais mortes por IAM do que naqueles com clima ameno, além de aumento na taxa de mortalidade por doenças cardíacas ${ }^{(6)}$. A poluição do ar e a temperatura são variáveis com maior influência no aumento de mortes por IAM. Pesquisadores apontam três motivos principais que podem explicar a relação entre as condições do tempo e as variações no número de infartos. Além das inflamações respiratórias que colaboram com problemas cardiovasculares, os fatores da coagulação do sangue ficam mais ativos em dias frios, favorecendo o fechamento das artérias coronárias e a formação de coágulos sangüíneos, fato demonstrado em estudos onde parâmetros hemostáticos estavam alterados, com exceção do TP(23, 21). Um terceiro motivo seria a vasoconstrição. Para evitar a perda de calor em baixas temperaturas, os vasos sangüíneos se contraem, provocando a elevação da pressão arterial e a obstrução em pessoas que já possuem algum tipo de placa de gordura que dificulte a circulação arterial|(21).

Verificou-se que baixos níveis de $\mathrm{Hb}$ estão associados a alta mortalidade em curto prazo de pacientes com IAM ${ }^{(11)}$. Uma terapêutica específica em pacientes anêmicos com IAM pode ser futuramente considerada. Neste estudo a média dos resultados de $\mathrm{Hb}$ não apresentou diferença significativa entre os grupos. Para o Ht, a média dos resultados obtidos também não apresentou diferença significativa entre os grupos (Tabela 1). No entanto, em pacientes com IAM o $\mathrm{Ht}$ é indicador para intervenções coronarianas. Num estudo observou-se que a presença de trombo intracoronariano estava relacionada significativamente com pacientes que apresentavam hematócrito alto(8).

A enzima gama-GT apresentou-se elevada em 52\% dos pacientes, sendo que a literatura mostra que apenas 5\%-30\% dos pacientes com IAM apresentam essa enzima com níveis sangüíneos elevados. O fato pode ser explicado devido a esses pacientes apresentarem problemas hepáticos, situação em que a enzima também estaria elevada ${ }^{(17)}$. 


\section{Conclusão}

O presente trabalho observou tendência de aumento crescente da concentração de fibrinogênio nos pacientes com IAM.

A definição de um perfil hemostático sugestivo de fatores de risco para desenvolver infarto seria altamente desejável, considerando o seu valor potencial para o diagnóstico precoce dessa grave doença, o que possibilitaria a adoção de medidas profiláticas. Uma comparação dos resultados dos parâmetros hemostáticos permite dizer que o encontro de níveis elevados de fibrinogênio assume grande importância na definição desse perfil nos pacientes estudados quando comparados aos controles. Portanto, os resultados encontrados suportam a relevância das alterações hemostáticas na fisiopatologia do IAM, considerando que a presença e a intensidade dessa alteração estão intimamente relacionadas com o agravamento da forma clínica.
Existem, nestes pacientes, alterações no tempo de protrombina, carecendo de estudos futuros para verificar a sua importância.

Os resultados mostram a presença de fatores de risco nos pacientes avaliados, como história familiar de DAC, tabagismo e sedentarismo.

Conclui-se que, também no nosso grupo de estudo, em dias com temperaturas baixas ou com extremos de temperatura aumentam os casos de IAM.

A contagem de leucócitos foi $50 \%$ maior em pacientes com IAM que no grupo controle, confirmando estudos anteriores que demonstram a ação inflamatória existente nesse processo.

A enzima gama-GT apresentou-se elevada nesses pacientes, necessitando de mais estudos para verificar sua colaboração para o diagnóstico do IAM.

Em nosso grupo de estudo verificou-se a importância da história familiar como fator de risco para IAM.

\section{Referências}

I. ANDREOLI,T.E. et al. Cardiopatia coronariana. In: CECIL. Medicina interna básica. 4 ed. Rio de Janeiro: Guanabara Koogan, 1998. p. 52-3.

2. BALLONE, G.J. Personalidade tipo A e cardiologia. In: Psiq-web, internet, 200 I. Disponível em: http://gballone.sites.vol.com. $\mathrm{br} / \mathrm{psicossomática/cardiologia3.htlm>2003.}$

3. DOMINGOS,A.E. Alterações climáticas e doenças cardiovasculares no município de Santa Gertrudez - SP. Rio Claro, 200I. Monografia - IGCE-UNESP.

4. FARKOUH, M.E. et al. A clinical trial of a chest pain observation unit for patients with unstable angina. N Engl J Med, v. 339, p. $1882-8,1998$.

5. HALPERN, A.; MANCINI, M.C. O tratamento da obesidade no paciente portador de hipertensão arterial. Rev Bras Hipertens, v. 7, p. 2, 2000.

6. HEUNIS, J.C.; OLIVIER, J.; BOURNE, D.E. The short-term relationships between winter temperatures and cardiac disease mortality in Cape Town. S Afr Med J, v. 85, n. I0, p. 1016-9, 1995.

7. HERRICK, J.B. Clinical features of sudden obstruction of the coronary arteries. JAMA, v. 59, p. 2015-20, 1912.

8. INOUE, F. et al. Hematocrit as an indicator for coronary intervention in patients with acute myocardial infarction. Am J Cardiol, v. 42, n. 6, p. 24I-7, 2003.

9. KOMAROV, A.L. et al. D-dimer and platelet aggregability are related to thrombotic events in patients with peripheral arterial occlusive disease. Eur Heart J, v. 23, p. 1309-16, 2002
10. KOSTIS, J.B.; BAUGHMAN, D.j; KUO, P.T. Association of recurrent myocardial infarction with haemostatic factors: a prospective study. Chest, v. 8I, n. 5, p. 57I-5, 1982.

I I. LIPSIC, E. et al. Hemoglobin levels and 30-day mortality in patients after myocardial infarction. Int J Cardiol, v. 100, p. 289-92, 2005.

12. LOWE, G.D.O. et al. Relationships of plasma viscosity, coagulation and fibrinolysis to coronary risk factors and angina. Thromb Haemost, v. 65, p. 339-43, 1991.

13. MAGALHÃES, C. et al.Avaliação do teste de triagem mistura |:| (plasma paciente: plasma normal) do TTPA nos resultados positivos do anticoagulante. Revista Brasileira de Hematologia e Hemoterapia temas livres/abstracts, ago. Supl. 2, p. I- | 88, 2003.

14. MURPHY, F.N.; STEWA, S.; MacINTYRE, K. Seasonal variation in morbidity and mortality related to atrial fibrillation. Int J Cardiol, v. 97, p. 283-8, 2004.

I 5. NOBIS, H. et al. Course and blood coagulation findings following systemic short-term fibrinolysis in acute myocardial infarct. Wien Klin Wochenschr, v. 98, n. 17, p. 573-7, 1986.

16. POPE, H. J. et al. Missed diagnoses of acute cardiac ischemia in the emergency department. $N$ Engl J Med, v. 342, p. 163-70, 2000.

17. RAVEL, R. Aplicações clínicas dos dados laboratoriais. In: Laboratório clínico. Rio de Janeiro: Ed. Guanabara, 1997.

18. RIDKER, P.M. et al. C-reactive protein and other markers of inflammation in the prediction of cardiovascular disease in women. N Engl J Med, v. 342, p. 836-43, 2000. 
19. ROBERTS, R.; KLEIMAN, N.S. Earlier diagnosis and treatment of acute myocardial infarction necessitates the need for a "new diagnostic mind-set". Circulation, v. 89, p. 872-8I, 1994.

20. ROSS, R. Aterosclerose. In: CECIL. Tratado de medicina interna. 20. ed. Rio de Janeiro: Guanabara Koogan, 1997. V. I, cap. 7; p. 323.

2I. SHAROVSKY, R.; CÉSAR, L.A.M.; RAMIRES, J.A.F.Temperature, air pollution, and mortality from myocardial infarction in São
Paulo, Brazil. Braz J Med Biol Res, v. 37, p. I65 I-7, 2004.

22. YAZBEK, P.j; BATTISTELLA, L.R. Álcool e coração. Revista da Sociedade de Cardiologia do Estado de São Paulo, v. 6, n. 4, p. 452-4, 1996

23. YEH, C.J.; CHAN, P.; PAN, W.H. Values of blood coagulating factors vary with ambient temperature: the cardiovascular disease risk factor two-township study in Taiwan. Clin J Physiol, v. 39, n. 2, p. $111-6,1996$. 